\title{
Is the model of one-armed oscillations able to explain the long-term V/R changes of Be stars?
}

\author{
R. Fiřt ${ }^{1,2}$ and P. Harmanec ${ }^{1,3}$ \\ 1 Astronomical Institute of the Charles University, V Holešovičkách 2, 18000 Praha 8, Czech Republic \\ 2 Mathematical Institute of the University of Bayreuth, Universitätsstraße 30, 95447 Bayreuth, Germany \\ e-mail: roman.firt@uni-bayreuth.de \\ 3 Astronomical Institute of the Academy of Sciences, 25165 Ondřejov, Czech Republic
}

Received 19 January 2005 / Accepted 28 September 2005

\section{ABSTRACT}

Context. Many scientists studying Be stars currently adopt the model of one-armed oscillations as the correct explanation of the cyclic longterm $V / R$ variations observed for a number of Be stars. We test the ability of this model to be used for the predictions of $V / R$ variations in real observed Be stars.

Aims. The behavior of the one-armed oscillations can be described as a solution of linearized hydrodynamical equations with the presence of "distorted" gravitational potential and a radiation force.

Methods. We developed a new computer program to model one-armed oscillations in Be star disks, which includes both the pressure force and the quadrupole term in the gravitational potential, related to the obliquity of a rapidly rotating star inside the disk. Moreover, we slightly improved the model in an effort to decrease the number of input parameters with the help of NLTE stellar atmosphere models.

Results. We carried out detailed tests of the dependence of $V / R$ "periods" predicted by the model on all individual input parameters. We arrived at the following results: (1) the model has great potential to explain not only the cause of the cyclic long-term $V / R$ changes but also some of the observed statistical properties of the phenomenon. (2) The model in its present linear form cannot be considered as proven. Its ability to predict the duration of $V / R$ cycles for individual well observed Be stars is insufficient. Changing some of the input parameters of the model, which are still loosely constrained by observations and/on current understanding of the disks, like the radial density distribution in the disk, one can easily arrive at any desired cycle length from, say, 1 to 20 years.

Conclusions. Clearly, a much more sophisticated non-linear and self-consistent model of disk structure and its oscillations will be needed before a truly quantitative test of a one-armed model vs. observations will be possible.

Key words. stars: emission line, $\mathrm{Be}$ - stars: oscillations - stars: circumstellar matter

\section{Introduction}

Be stars belong to the most variable objects in the upper part of the main sequence belt in the HR diagram. A well recognized type of long-term spectral variation are cyclic variations in the relative intensities of the Violet and Red component of double-peaked Balmer emission lines $(V / R$ variations hereafter) (McLaughlin 1961). The quasi-periods of these changes range from a few years to more than a decade and are usually also accompanied by simultaneous radial-velocity variations of the whole emission-line profile. McLaughlin (1961) demonstrated that the variations cannot be caused by any type of radial oscillations in the disk and put forward the hypothesis, originally outlined by Struve (1931), that they are related to a gradual revolution of an elongated (elliptical) disk. As pointed out by Johnson (1958), a simple explanation of why such a disk revolves is an analogy to apsidal motion in a binary system.
Quantitative modelling of $V / R$ changes are discussed in e.g., Harmanec (1982, 2001); Mennickent et al. (1997) and references given there. Here, we shall concentrate on one particular explanation of the long-term $V / R$ variations - the model of one-armed non-radial oscillations in Be star disks.

Following the suggestions of Kato (1983), Okazaki (1991) constructed a model in which the $V / R$ variations are caused by global non-radial one-armed oscillation in the equatorial disk. The only mechanism that governs the one-armed oscillation in his model was the pressure force. In order to make the oscillation periods finite (they turned out to grow to infinity with the increasing radius of the disk), Okazaki (1991) had to restrict the outer radius of the disk to a finite value. He indeed found that oscillation periods of the order of several years can be obtained from the model. However, in the vast majority of cases, the one-armed density wave has a retrograde motion with respect to the direction of the stellar and disk rotation. This was in contradiction to the existing observational evidence of 
prograde revolution of the density enhancement (Telting et al. 1994; Mennickent et al. 1997; Vakili et al. 1998; Berio et al. 1999).

To overcome this shortcoming of Okazaki's model, Papaloizou et al. (1992) demonstrated that the one-armed density waves are only confined to the inner parts of the disk if the quadrupole term is introduced to the gravitational potential. This term naturally arises from a rotationally induced distortion (flattening) of a rapidly rotating star inside the disk. Savonije \& Heemskerk (1993) constructed a model based on this idea and obtained prograde revolution of density waves with reasonable periods.

However - as pointed out by Okazaki (1996) - the effect of the rotational distortion of the star was overestimated in their model while the radiation effect was completely neglected. Okazaki also argued that the temperatures of the disks they used were too low to apply to real Be stars. As an alternative, Okazaki (1996) constructed a new model that includes both the radiative pressure and rotational distortion of the star. However, the price to be paid was the increase in the number of input parameters. This, of course, has an adverse effect on the uniqueness of the model. The more parameters that can be tuned in the model, the easier it is to match the observations.

The purpose of this study is twofold.

First, we attempt to reduce the number of free input parameters using the NLTE stellar atmosphere models. In Sect. 2 we briefly describe the hydrodynamical model used in our calculations. We describe the basic assumptions. For more details, readers are referred to Okazaki $(1996,1997)$ and references therein. A brief description of the numerical method and the boundary conditions used can be found in Sect. 3. Our approach is similar to that by Savonije \& Heemskerk (1993).

Second, we investigated the dependence of the duration of the $V / R$ cycles predicted by the model on all individual input parameters entering the model. These tests are presented in Sect. 7. They corroborate the warning expressed by Gayley et al. (2001) that the one-armed oscillation model of longterm $V / R$ variations of $\mathrm{Be}$ stars cannot be considered as proven, as it is often presented in many recent Be-star studies.

\section{The model of the disk}

We assume the unperturbed disk to be geometrically thin, axisymmetric and isothermal. It consists of inviscid compressible fluid. The temperature of the disk $T_{\mathrm{d}}$ satisfies the following condition

$$
0.5 \leq \frac{T_{\mathrm{d}}}{T_{\mathrm{eff}}} \leq 1
$$

where $T_{\text {eff }}$ is the effective temperature of the central star. The gas is assumed to obey the polytropic relation between pressure $p$ and density $\rho$

$$
p \propto \rho^{\gamma}
$$

with $\gamma=1$.
Following Papaloizou et al. (1992); Savonije \& Heemskerk (1993); Okazaki (1991), we approximate the gravitational potential of the central star as

$\Psi(r, z) \approx-\frac{G M}{\sqrt{r^{2}+z^{2}}}\left[1+\frac{k_{2} f^{2}}{3} \frac{R^{2}}{r^{2}+z^{2}}\left(1-\frac{3 z^{2}}{r^{2}+z^{2}}\right)\right]$,

where $G$ denotes the gravitational constant, $M$ and $R$ mean mass and radius of the star, $k_{2}$ being the so-called apsidalmotion constant (see Schwarzschild 1958) and $f$ is the parameter which characterizes the stellar rotation. It is defined as the ratio of the true to critical (Keplerian) rotational velocity $\Omega_{\mathrm{K}}$ at the stellar surface:

$f=\frac{\Omega(R)}{\Omega_{\mathrm{K}}(R)}$.

We use a cylindrical coordinate system $(r, \varphi, z)$ with the origin placed at the center of the star and with $z$-axis perpendicular to the disk equatorial plane. The inner radius of the disk $r_{\text {in }}$ is set equal to the equatorial radius of the star. The outer radius of the disk $r_{\text {out }}$ is assumed to satisfy the following relation

$r_{\text {out }}=\xi r_{\text {in }}$,

where $\xi$ is one of the free input parameters of the problem.

We impose the $m=1$ nonradial mode perturbations of velocity field $v$, pressure and density on an unperturbed disk in the following way:

$$
\begin{aligned}
v_{r} & =v_{r}^{\prime}(r, \varphi, z, t), \\
v_{\varphi} & =r \Omega(r)+v_{\varphi}^{\prime}(r, \varphi, z, t), \\
v_{z} & =v_{z}^{\prime}(r, \varphi, z, t), \\
\rho & =\rho_{0}(r, z)+\rho_{1}(r, \varphi, z, t), \\
p & =p_{0}(r, z)+\underbrace{c_{\mathrm{s}}^{2} \rho_{1}(r, \varphi, z, t)}_{\equiv p_{1}(r, \varphi, z, t)} .
\end{aligned}
$$

The governing equations of this model could be written after a proper linearization in the perturbed quantities as follows (from now on the symbols of the perturbed quantities denote only their radial parts) (see Okazaki 1991, 1996)

$$
\begin{aligned}
& \mathrm{i}(\sigma-\Omega) v_{r}^{\prime}-2 \Omega v_{\varphi}^{\prime}=-c_{\mathrm{s}}^{2} \frac{\mathrm{d}}{\mathrm{d} r}\left(\frac{\rho_{1}}{\rho_{0}}\right), \\
& \mathrm{i}(\sigma-\Omega) v_{\varphi}^{\prime}+\frac{\kappa}{2 \Omega} v_{r}^{\prime}=\mathrm{i} \frac{c_{\mathrm{s}}^{2}}{r} \frac{\rho_{1}}{\rho_{0}}, \\
& \mathrm{i}(\sigma-\Omega) \frac{\rho_{1}}{\rho_{0}}+\frac{1}{r \sigma_{0}} \frac{\mathrm{d}}{\mathrm{d} r}\left(r \sigma_{0} v_{r}^{\prime}\right)-\frac{\mathrm{i} v_{\varphi}^{\prime}}{r}=0 .
\end{aligned}
$$

Here $c_{\mathrm{s}}$ denotes the local speed of sound and $\Omega$ is the angular frequency of the unperturbed flow which can be extracted (under approximation $z \ll r$ ) from the condition of balance of all relevant forces in the disk (i.e. the centrifugal, gas pressure and radiative force $F_{\text {rad }}$ )

$r \Omega^{2}-\frac{1}{\rho_{0}} \frac{\partial p_{0}}{\partial r}-\frac{\partial \Psi}{\partial r}+F_{\mathrm{rad}} \frac{r}{\sqrt{r^{2}+z^{2}}}=0$

as

$\Omega(r) \simeq \Omega_{\mathrm{K}}(r)\left[1-\alpha \mathcal{M}_{\mathrm{s}}^{-2} \frac{r}{R}+k_{2} f^{2}\left(\frac{r}{R}\right)^{-2}-\eta\left(\frac{r}{R}\right)^{\varepsilon}\right]^{\frac{1}{2}}$, 
where $\mathcal{M}_{\mathrm{s}}$ denotes the Mach number of the flow at the stellar surface. It is connected with the disk temperature to the following way:

$\mathcal{M}_{\mathrm{s}}=\sqrt{\frac{G M m_{\mathrm{p}}}{R k_{\mathrm{B}} T_{\mathrm{eff}}}}\left(\frac{T_{\mathrm{d}}}{T_{\mathrm{eff}}}\right)^{-\frac{1}{2}}$,

where $k_{\mathrm{B}}$ denotes the Boltzmann's constant and $m_{\mathrm{p}}$ is the weight of the disk's particles. Following Okazaki (1997) we have adopted the mean molecular weight of the particles to be 0.6. In the present model, the epicyclic frequency $\kappa$ is given (again under assumption $z \ll r$ ) by the expression

$\kappa(r) \simeq \Omega_{\mathrm{K}}\left[1-2 \alpha \mathcal{M}_{\mathrm{s}}^{-2} \frac{r}{R}-k_{2} f^{2}\left(\frac{r}{R}\right)^{-2}-\eta(1+\varepsilon)\left(\frac{r}{R}\right)^{\varepsilon}\right]^{\frac{1}{2}}$.

To obtain the unperturbed density $\rho_{0}$ we use the assumption that the disk is in hydrostatic equilibrium in the $z$-direction which yields the expression

$\rho_{0}(r, z)=\rho_{00}(r) \exp \left(-\frac{z^{2}}{2 H^{2}}\right)$,

where $\rho_{00}$ denotes the density distribution in the equatorial plane. Since these profiles are not well constrained from existing observations, we simply put

$\rho_{00}(r) \propto\left(\frac{r}{R}\right)^{-\alpha}$.

$H$ denotes the vertical scale height of the disk given by

$H(r)=\frac{c_{\mathrm{s}}}{\Omega_{\mathrm{K}}}\left[1+3 k_{2} f^{2}\left(\frac{r}{R}\right)^{-2}-\eta\left(\frac{r}{R}\right)^{\varepsilon}\right]^{-\frac{1}{2}}$.

Index $\alpha$ in (13) is another free parameter of the problem. The radiative force $F_{\text {rad }}$ and the parameters $\eta$ and $\varepsilon$ are discussed in more detail in Sect. 5.

\section{Numerical method and the boundary conditions}

Eliminating $v_{\varphi}^{\prime}$ from equations (7), one obtains the following set of ordinary differential equations:

$$
\begin{aligned}
\frac{\mathrm{d}}{\mathrm{d} r}\left(\frac{\rho_{1}}{\rho_{0}}\right) & =\frac{2 \Omega}{r(\sigma-\Omega)} \frac{\rho_{1}}{\rho_{0}}-\frac{(\sigma-\Omega)^{2}-\kappa^{2}}{(\sigma-\Omega) c_{\mathrm{s}}^{2}} \mathrm{i} v_{r}^{\prime}, \\
\mathrm{i} \frac{\mathrm{d}}{\mathrm{d} r} v_{r}^{\prime}= & {\left[\sigma-\Omega-\frac{c_{\mathrm{s}^{2}}}{r^{2}(\sigma-\Omega)}\right] \frac{\rho_{1}}{\rho_{0}} } \\
& -\left[\frac{\kappa^{2}}{2 r \Omega(\sigma-\Omega)}+\frac{\mathrm{d}}{\mathrm{d} r} \ln \left(r \sigma_{0}\right)\right] \mathrm{i} v_{r}^{\prime} .
\end{aligned}
$$

These equations can be solved numerically if some reasonable boundary conditions are set. We followed the same numerical method as Savonije \& Heemskerk (1993), the so-called shooting to a fitting point method. We integrate the equations from both boundaries up to some chosen fitting point and iterate the free variables until they match the boundary conditions and the continuity condition at the fitting point. A more detailed description of the method can be found in Press et al. (1992). During the numerical calculations, we used normalized quantities assuming the following units: $G=1, M=1$ and $R=1$.
We adopted the following boundary conditions: we assume the stellar surface to be rigid and not penetrating into the disk. At the same time, we assume that the disk is not geometrically detached from the star. This implies the following condition at the inner radius of the disk:

$v_{r}^{\prime}\left(r_{\text {in }}\right)=0$

The choice of the outer boundary condition is less obvious. As already mentioned in Sect. 1, one cannot expect that the volume of the disk in which the observed Balmer emissions originate is identical to the whole volume occupied by the disk. Instead, we assume that there is some small but non-zero pressure $p_{\text {ext }}$ in the outer parts of the disk at some large distance from the central star $r_{\text {out }}$ and that this pressure is no longer changing with the increasing distance. An equivalent of this condition is that for each particle at the outer radius, the Lagrangian (material) time derivative of the pressure will be zero:

$\dot{\bar{p}}=\frac{\partial p}{\partial t}+\boldsymbol{v} \cdot \nabla p=0 \quad$ at $\quad r=r_{\text {out }}$.

This condition could be rewritten after linearization as

$v_{r}^{\prime}-i(\sigma-\Omega) \frac{\rho_{1}}{\rho_{0} \frac{\mathrm{d} \ln \sigma_{0}}{\mathrm{~d} r}}=0 \quad$ at $\quad r=r_{\text {out }}$.

Here $\sigma_{0}$ is the total surface density defined by

$\sigma_{0}(r)=\int_{-\infty}^{+\infty} \rho_{0} \mathrm{~d} z=\sqrt{2 \pi} \rho_{00}(r) H(r)$

\section{The reference model}

The model of one-armed oscillations in the disk, formulated in the previous two sections, has as many as 9 free parameters:

- $M-$ stellar mass;

- $R$-stellar radius;

- $T_{\mathrm{d}}$ - temperature of the disk;

- $f$ - stellar rotational parameter defined as the ratio of true to Keplerian rotational velocity at the stellar surface (see Eq. (4));

- $k_{2}$ - internal structure constant affecting the rate of apsidal motion;

- $\xi$ - outer disk radius in the units of the inner radius of the disk (assumed here to be equal to the stellar equatorial radius) (see Eq. (5));

- $\alpha-$ index of the equatorial density profile (see Eq. (13));

- $\eta, \varepsilon$ - parameters characterizing the radiative field (see Sect. 5).

It is true that the above 9 parameters are not completely independent of each other. For a given stellar mass, the stellar radius and the constant of internal structure are defined by the evolutionary stage of the star. However, the evolutionary stage of particular Be stars is usually unknown and the one-to-one relation between the stellar mass and radius need not be preserved for rotating stars. The internal structure constant for rapidly rotating bodies is not well known either. There is another constraint 
Table 1. The input parameters of the reference model.

\begin{tabular}{lclclc}
\hline \hline$M$ & $7.94 M_{\odot}$ & $f$ & 0.95 & $\alpha$ & 3.5 \\
$R$ & $4.59 R_{\odot}$ & $k_{2}$ & 0.007 & $\eta$ & 0.011 \\
$T_{\text {eff }}$ & $20800 \mathrm{~K}$ & $\xi$ & 40 & $\varepsilon$ & 0.09 \\
\hline
\end{tabular}

for Be stars being members of binary systems. The outer radius of the disk cannot exceed the dimensions of the respective Roche lobe for them which sets a natural limit on parameter $\xi$.

It is therefore of interest to investigate how the period of the one-armed oscillation predicted by the model depends on each individual input parameter. To this end, we first define the reference model with respect to which we vary individual parameters of the model to see their effect on the period of the one-armed mode. Considering that the highest percentage of Be stars is found for stars of spectral subclass B2 (Slettebak 1982), we have chosen a model star of spectral class B2 for our reference model. Its main characteristics, defining the reference model, are given in Table 1 . To proceed in a consistent way, we adopted the stellar mass of $8 M_{\odot}$ and all other parameters corresponding to its evolutionary model calculated by Claret (2004) for the age at half of its main-sequence life. It was demonstrated by Harmanec (2003) that the basic properties of stellar interior models for this choice very nicely agree with the empirical calibration of masses and radii as functions of the effective temperature, based on accurate data from eclipsing binaries, as derived by Harmanec (1988). Following Okazaki (1997), we set the disk temperature $T_{\mathrm{d}} \sim \frac{2}{3} T_{\text {eff }}$. According to Waters et al. (1987), the index of the equatorial density profile $\alpha$ falls into a range $2 \lesssim \alpha \lesssim 3.5$ while Okazaki (1997) suggests a range $2.5 \lesssim \alpha \lesssim 4$.0. Following Okazaki, we adopt $\alpha=3.5$ which according to him corresponds to the theoretical value for a subsonic region of an isothermal disk. The stellar rotational parameter $f$ was chosen close to break-up at the equator, in the accordance with the recent studies of particular well observed Be stars by Harmanec et al. (2002); Harmanec (2002, 2003) as well as theoretical arguments by Townsend et al. (2004); Frémat et al. (2005). The outer radius of the disk, $\xi R$, was chosen large enough not to interfere with the confined mode. In such a case, the resulting oscillation period depends only weakly on the specific value of $\xi$.

\section{Radiative force}

Let us discuss now the radiative force which - according to Okazaki (1997) - plays a decisive role in the $V / R$ phenomenon for early $B$ stars. In his model, Okazaki calculates the radiative force arising from an ensemble of optically thin lines, $F_{\text {rad }}$, with the help of an empirical formula derived by Chen \& Marlborough (1994):

$F_{\mathrm{rad}}(r, z) \approx \frac{G M}{r^{2}+z^{2}} \eta\left(\frac{r^{2}+z^{2}}{R^{2}}\right)^{\frac{\varepsilon}{2}}$.

According to Chen \& Marlborough (1994), the parameters $\eta$ and $\varepsilon$ which determine the strength of the force are both positive numbers, much smaller then 1 , but their exact values are not clearly constrained by the theory. They simply represent free parameters to be tuned for the given problem.

The assumption that only the optically thin lines are responsible for the radiative force is actually quite limiting since this implies exclusion of the presence of any optically thick lines. Note that possible effects of optically thick lines on the behavior of Be-star disks have been studied in detail by Gayley et al. (2001).

To address this problem, and also in an effort to decrease the number of free parameters of the model we chose to modify Okazaki's approach as follows. We adopted the profile of the force given by Eq. (21) from Chen \& Marlborough (1994) but assumed that the force arises from both optically thin and thick lines. The next task is to calculate the radiative parameters $\eta$ and $\varepsilon$. If one rewrites Eq. (21) in the units which we use in the calculation and evaluates the force at the stellar surface, one obtains

$F_{\text {rad }}(R)=\eta$.

If one uses a model atmosphere, it is possible to calculate the value of the total radiative force over the whole electromagnetic spectrum at the surface of the central star and use it to evaluate the value of $\eta$ explicitly. To this end, we used program TLUSTY developed by Hubený (1988) which allows to compute NLTE stellar atmosphere models of the stars of different effective temperatures, surface gravities and chemical compositions. For our purpose, we simply assumed solar composition in all calculations. Gravity was derived from the mass and radius of each considered model.

\section{A trial application to two well-known Be binaries}

One way to investigate the applicability of the model of onearmed oscillations to real Be stars is to apply it to objects for which one has some idea about as many of the input parameters of the model as possible. For that reason we have chosen two well-known Be stars that are members of binary systems, $\gamma$ Cas = HD 5394 and 59 Cyg = V832 Cyg. Their most probable basic physical properties were recently derived by Harmanec (2002) and Harmanec et al. (2002), respectively. An apparent advantage of choosing Be stars in binary systems is that one can take the value of parameter $\xi$ in such a way that the upper limit to $\xi R$ is given by the dimensions of the corresponding Roche lobe around the Be star in question ${ }^{1}$. However the application of the model in its present form to a binary system has serious shortcomings. There are several related problems:

1. The tidal potential from the companion represents a new parameter of the problem and should be properly modelled. The outer part of the disk becomes a propagation region, making the confinement of the mode to the inner part of the disk less efficient.

2. The radius of the disk might become tidally truncated due to resonance. However the direct evidence from

\footnotetext{
1 Note that the relative dimensions of the Roche lobe are only given by the mass ratio and the absolute ones depend only very weakly on the less safely constrained orbital inclination for both binaries.
} 
Table 2. Input parameters of the model of $\gamma$ Cas.

\begin{tabular}{lcl}
\hline \hline Parameter & Value & Source \\
\hline$T_{\text {eff }}$ & $33000 \mathrm{~K}$ & Hutchings (1970); Harmanec (2002) \\
$\log g$ & 3.89 & Harmanec (2002) \\
$M$ & $18.3 M_{\odot}$ & Harmanec (2002) \\
$R$ & $8.0 R_{\odot}$ & Harmanec (2002) \\
$T_{\mathrm{d}}$ & $22000 \mathrm{~K}$ & Okazaki (1997) \\
$f$ & 0.9 & Townsend et al. (2004) \\
$k_{2}$ & 0.008 & Claret (2004) \\
$\xi$ & 22.5 & Roche-lobe limit \\
$\alpha$ & 3.5 & Okazaki (1997) \\
\hline
\end{tabular}

Table 3. Input parameters of the model of 59 Cyg.

\begin{tabular}{lcl}
\hline \hline Parameter & Value & Source \\
\hline$T_{\text {eff }}$ & $26000 \mathrm{~K}$ & Harmanec et al. (2002) \\
$\log g$ & 4.099 & Harmanec et al. (2002) \\
$M$ & $10.78 M_{\odot}$ & Harmanec et al. (2002) \\
$R$ & $4.85 R_{\odot}$ & Harmanec et al. (2002) \\
$T_{\mathrm{d}}$ & $17333 \mathrm{~K}$ & Okazaki (1997) \\
$f$ & 0.9 & Townsend et al. (2004) \\
$k_{2}$ & 0.009 & Claret (2004) \\
$\xi$ & 10.5 & Roche-lobe limit \\
$\alpha$ & 3.5 & Okazaki (1997) \\
\hline
\end{tabular}

Tycner et al. (2004) and indirect evidence for some other known Be stars in binaries discussed by Harmanec (2003) (radial velocity of emission wings indicate that the inner parts of the disks move in orbit with the primary while the phase-locked $V / R$ variations show that the outer parts of the disk must be elongated towards the secondary) seem to indicate that a large part of available volume inside the Roche-lobe radius is occupied by the disk.

3. The one-armed modes need not be confined to the inner parts of such disks and even retrograde modes can develop in such a situation.

Considering all this, the modelling presented in this section should only be understood as an illustrative.

The particular values of the input parameters which we adopt are listed in Tables 2 and 3 for $\gamma$ Cas and 59 Cyg, respectively. Note that - since we do not know the exact evolutionary stage of both Be stars - we adopt approximate values of the constant $k_{2}$ for both stars, close to the values corresponding to their masses, from evolutionary models by Claret (2004). The corresponding values of $\eta$ calculated with TLUSTY are

$\eta_{\gamma \mathrm{Cas}} \simeq 0.088$,

$\eta_{59 \mathrm{Cyg}} \simeq 0.021$.

The individual observed $V / R$ cycles of $\gamma$ Cas range from about 3 to 5 years while a rather stable 2-year cycle was observed

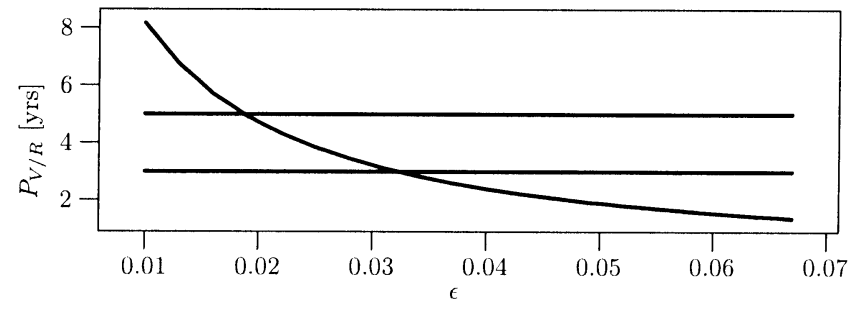

Fig. 1. The period of one-armed oscillation as a function of radiative parameter $\varepsilon$ for the model of $\gamma$ Cas. The horizontal lines define the range of the lengths of individual $V / R$ cycles observed for this star.

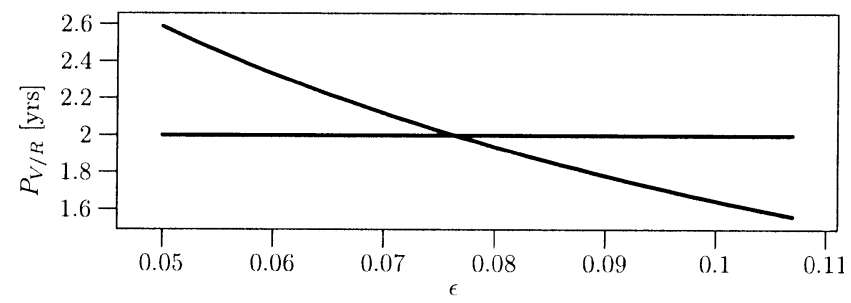

Fig. 2. The period of one-armed oscillation as a function of radiative parameter $\varepsilon$ for the model of $59 \mathrm{Cyg}$. The horizontal line corresponds to the length of individual $V / R$ cycles observed for this star.

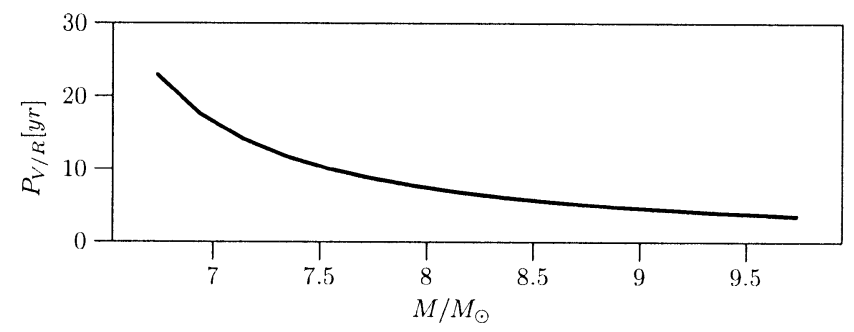

Fig. 3. The dependence of the $V / R$ cycle length on the stellar mass $M$.

for 59 Cyg. The periods of one-armed oscillations predicted by the model calculations, based on the input parameters given in Tables 2 and 3, are plotted as functions of the input radiative parameter $\varepsilon$ in Figs. 1 and 2 for $\gamma$ Cas and 59 Cyg, respectively. The horizontal lines in both figures show the (range) of the observed lengths of $V / R$ cycles for the two stars.

One can see that to achieve agreement between the model and observed length of the $V / R$ cycles, one has to assume values of $\varepsilon$ of about 0.025 for $\gamma$ Cas and 0.075 for $59 \mathrm{Cyg}$.

\section{Tests of dependence of one-armed mode periods on individual input parameters}

To investigate the dependence of the cycle length of individual input parameters of the model, we started with the reference model defined in Sect. 4 and varied the free parameters, one by one, over reasonable ranges of their respective values. The results of our calculations are shown in Figs. 3-9.

Several comments are appropriate.

1. Our exercise clearly shows that varying any of the 8 free parameters lead to widely different theoretical cycle lengths, ranging at least from 1 to more than 10 years. In other words, tuning any one of the free parameters of the model, 


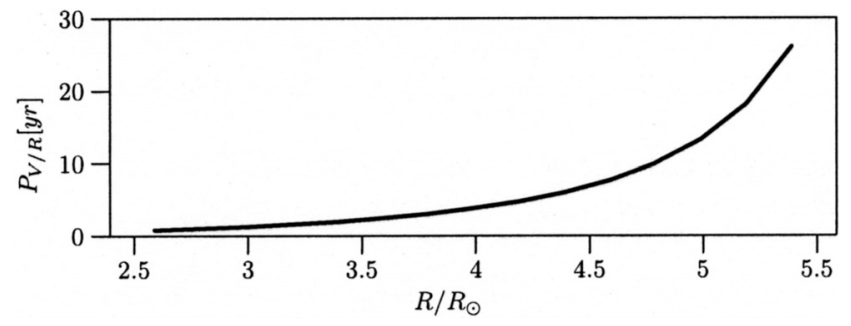

Fig. 4. The dependence of the $V / R$ cycle length on the stellar radius $R$.

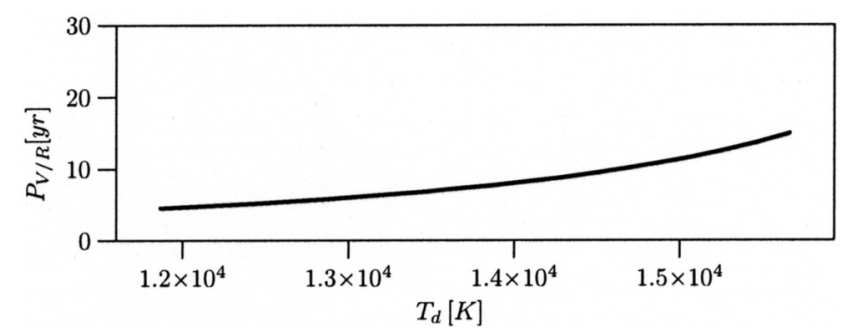

Fig. 5. The dependence of the $V / R$ cycle length on the disk temperature $T_{\mathrm{d}}$.

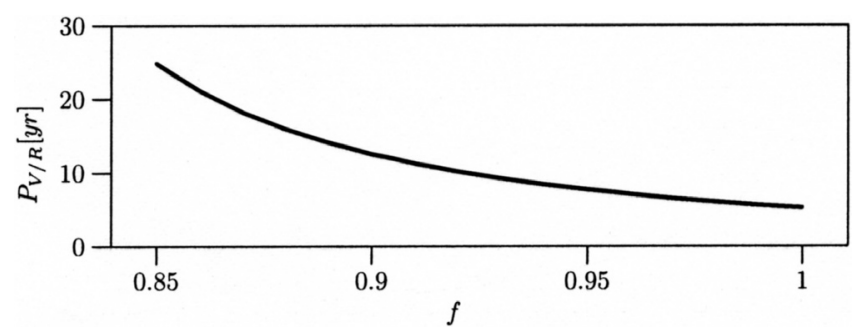

Fig. 6. The dependence of the $V / R$ cycle length on the ratio of the true to the critical rotational velocity at the equator $f$.

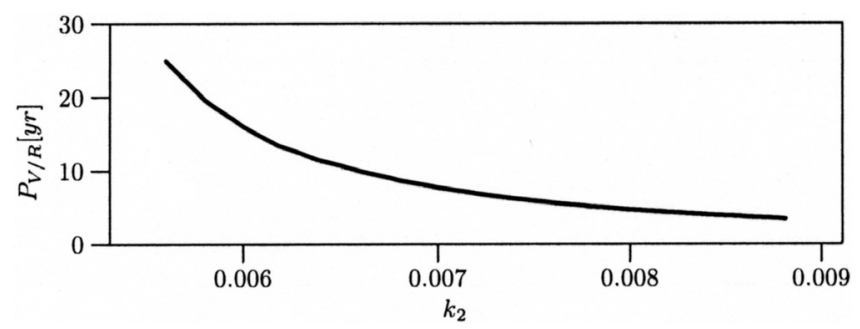

Fig. 7. The dependence of the $V / R$ cycle length on the constant of internal structure $k_{2}$.

one can reproduce the observed range of cycle lengths for all so far known $V / R$ Be variables.

2. One can, of course, set some limits on some of the free parameters in particular cases, as we did in Sect. 6, but even in the most favourable cases one is left with two largely unconstrained parameters, the radiative parameter $\varepsilon$ and parameter $\alpha$ which describes the density distribution in the disk. As Fig. 8 shows, tuning $\alpha$ alone over the range of probable values allows one to achieve agreement with any observed cycle length from 3 to 20 years (for the particular case of our reference model). This implies that - until the density distribution of the disks will directly be observed or predicted on the basis of self-consistent physical models - the correctness of the model of one-armed oscillations

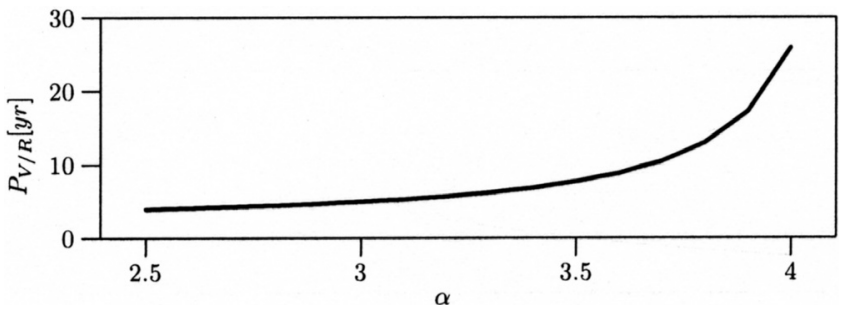

Fig. 8. The dependence of the $V / R$ cycle length on the coefficient characterizing the density distribution in the disk $\alpha$.

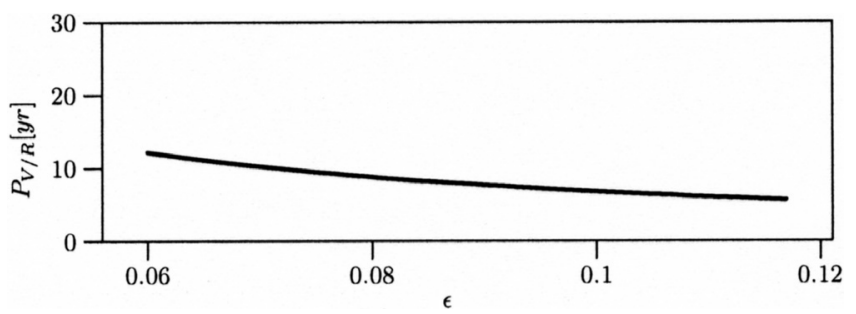

Fig. 9. The dependence of the $V / R$ cycle length on the radiative parameter $\varepsilon$.

cannot be seriously tested with existing observations. A similar problem is related to the value of the constant of interval structure $k_{2}$ since even the most recent models by Claret (2004) are derived for non-rotating stellar models. To the best of our knowledge, there is no published grid of rotating stellar models with a tabulation of internal structure constants. As Fig. 7 indicates, the inclusion of an appropriate constant for rotating stars may also have significant impact on the resulting cycle length of one-armed oscillations.

3. However, our exercise also shows great potential of the model. Note in particular the opposite dependence of the theoretical cycle lengths on the stellar mass and radius. This fact may explain the observed and somewhat surprising fact that the mean observed cycle lengths do not depend on the spectral subclass of the underlying stars (Copeland \& Heard 1963; Mennickent \& Vogt 1991).

To test such a possibility, we calculated theoretical $V / R$ cycles for mid-main sequence Claret (2004) models of B stars. The temperature of the disk $T_{\mathrm{d}}$ was again set to twothirds of the stellar effective temperature and the density distribution, fraction of break-up rotation rate and radiative parameters were set equal to those of the reference model since their behavior along the main sequence is not really known. The results are summarized in Table 4 where also the values adopted for the above-mentioned three parameters are given. One can see that our expectation was not fulfilled. The length of the $V / R$ cycles derived under such assumptions increases rapidly with the decreasing mass of the central star. Inspecting Fig. 4 in Okazaki (1997), we conclude that some increase of the observed $V / R$ cycles with the decreasing mass (and effective temperature) of central stars might indeed be observed but it is much less pronounced than what our modelling shows. There are a number of possible explanations like less rapid rotation of central stars or variation of the disk structure and radiative parameter along the $\mathrm{B}$ sequence but the exercise once more 
Table 4. The theoretical $V / R$ cycles calculated for Claret (2004) stellar interior models of $\mathrm{B}$ stars corresponding to an average age between the zero-age and terminal-age main sequence stage. In all cases, we kept $\alpha=3.5, f=0.95$ and $\varepsilon=0.09$ fixed.

\begin{tabular}{rrrccc}
\hline \hline $\begin{array}{r}M \\
\left(M_{\odot}\right)\end{array}$ & $\begin{array}{c}R \\
\left(R_{\odot}\right)\end{array}$ & $\begin{array}{r}T_{\text {eff }} \\
(\mathrm{K})\end{array}$ & $k_{2}$ & $\eta$ & $\begin{array}{c}P \\
(\mathrm{yrs})\end{array}$ \\
\hline 2.51 & 2.39 & 9800 & 0.004 & 0.002 & $>100$ \\
3.16 & 2.76 & 11500 & 0.004 & 0.002 & $>100$ \\
3.98 & 3.01 & 13700 & 0.005 & 0.002 & $>100$ \\
5.01 & 3.45 & 15900 & 0.005 & 0.003 & $>100$ \\
6.31 & 4.00 & 18200 & 0.006 & 0.006 & 44.29 \\
7.94 & 4.59 & 20800 & 0.007 & 0.011 & 7.69 \\
10.00 & 5.26 & 23600 & 0.008 & 0.018 & 5.12 \\
12.59 & 5.88 & 26600 & 0.008 & 0.029 & 2.31 \\
15.85 & 6.74 & 29400 & 0.008 & 0.046 & 1.54 \\
\hline
\end{tabular}

shows the insufficient predictive power of the one-arm oscillation model in its present formulation.

4. Directly related to this is the question of the real importance of the radiative force and the reality of the so called hybrid scenario advocated by Okazaki (1997). What he suggests is that while the radiative forces dominate the $V / R$ changes for the early-B stars, the oblateness of central stars is more important for the late-type stars. In his Fig. 5, Okazaki (1997) shows that according to his modelling, the $V / R$ cycle length attains a maximum for mid-B spectral subclasses and decreases both, for early-B and late-B stars. He implies that this is supported by really observed cycle lengths plotted vs. spectral subclass in his Fig. 4. However, as already mentioned, we rather see (if anything) a mild increase of the cycle length towards late B subclasses in that Figure. Nevertheless, it is fair to admit that there is still a large uncertainty in our knowledge of how close to the break-up real Be stars rotate. The closer to break-up, the more dominant is the effect of stellar oblateness over the effect of radiative forces and vice versa. We are convinced however that Okazaki's adoption of $f$ of 0.4 is unrealistically low. This in turn means that he overestimates the effect of radiative forces in his modelling. We agree, however, that the effect must be almost completely suppressed for the lateB stars. For these objects, the predictive power of the onearm model is certainly better than for early-B stars since the only free parameters, unconstrained by observations, are $\alpha$ and $k_{2}$ (if radiative forces have negligible effect).

\section{Conclusion}

We eliminated one of the free parameters of the model of onearmed disk oscillations which was suggested to explain the observed long-term cyclic $V / R$ changes of Be stars. We demonstrated that the model has great potential but is far from being proven. Its predictive power is very low due to a strong sensitivity of predicted cycle lengths on small changes in any of the eight physical parameters that define the model. The situation is slightly better (but still not satisfactory) for Be stars of later subclasses where the radiative forces play a negligible role and for Be stars in binaries where the outer radius of the disk is given by the corresponding Roche lobe. Note, however, that the high sensitivity to the physical parameters indicates a way to explain the non-stationarity of the phenomenon, i.e. why different $V / R$ cycles usually have different cycle length and why the phenomenon comes and go in some well observed Be stars.

Two very important steps are necessary to better understand the long-term $V / R$ changes:

1. To derive the true density and temperature structure of the disks, either via spectro-interferometric observations or from self-consistent models of the disks which would include radiative transfer. The latter approach would also allow a direct calculation of the radiative forces in the disk.

2. To abandon the current linear and stationary models in favour of non-linear 3D modelling.

3. Pay special attention to Be stars in binaries since the presence of the companion not only sets a limit to the outer dimension of the disk but can also affect the rate of the apsidal advance of the one-armed wave as pointed out by Kř́̌ž \& Harmanec (1975).

Acknowledgements. We would like to thank I. Hubený for his useful remarks on the NLTE stellar atmosphere model. Our special thanks go to the referee, Dr. A.T. Okazaki, for his helpful and constructive criticism of the first version of the study. This research was supported from the grant GA ČR 205/2003/0788 of the Granting Agency of the Czech Republic. The research of PH was also supported from the research project AV0Z10030501 of the Academy of Sciences of the Czech Republic. We acknowledge the use of the electronic bibliography maintained by NASA/ADS.

\section{References}

Berio, P., Stee, P., Vakili, F., et al. 1999, A\&A, 345, 203

Chen, H., \& Marlborough, J. M. 1994, ApJ, 427, 1005

Claret, A. 2004, A\&A, 424, 919

Copeland, J. A., \& Heard, J. F. 1963, Publications of the David Dunlap Observatory, 2, 317

Frémat, Y., Zorec, J., Hubert, A.-M., \& Floquet, M. 2005, A\&A, 440, 305

Gayley, K. G., Ignace, R., \& Owocki, S. P. 2001, ApJ, 558, 802

Harmanec, P. 1982, in Be Stars, IAU Symp., 98, 279

Harmanec, P. 1988, Bulletin of the Astronomical Institutes of Czechoslovakia, 39, 329

Harmanec, P. 2001, Publications of the Astronomical Institute of the Academy of Sciences of the Czech Republic, 89, 9

Harmanec, P. 2002, in Exotic Stars as Challenges to Evolution, ASP Conf. Ser., 279, 221

Harmanec, P. 2003, in Publ. Canakkale Onsekiz Mart Univ, New Directions for Close Binary Studies: The Royal Road to the Stars, 3,221

Harmanec, P., Božić, H., Percy, J. R., et al. 2002, A\&A, 387, 580

Hubený, I. 1988, Comp. Phys. Com., 52, 103

Hutchings, J. B. 1970, MNRAS, 150, 55

Johnson, M. 1958, Mem. Soc. Royale Sci. Liège IV. Ser., 20, 219 
Kato, S. 1983, PASJ, 35, 249

Kř́ž, S., \& Harmanec, P. 1975, BAICz, 26, 65

McLaughlin, D. B. 1961, JRASC, 55, 73

Mennickent, R. E., Sterken, C., \& Vogt, N. 1997, A\&A, 326, 1167

Mennickent, R. E., \& Vogt, N. 1991, A\&A, 241, 159

Okazaki, A. T. 1991, PASJ, 43, 75

Okazaki, A. T. 1996, PASJ, 48, 305

Okazaki, A. T. 1997, A\&A, 318, 548

Papaloizou, J. C., Savonije, G. J., \& Henrichs, H. F. 1992, A\&A, 265, L45

Press, W. H., Teukolsky, S. A., Vetterling, W. T., \& Flannery, B. P. 1992, Numerical recipes in FORTRAN (2nd Ed.): the art of scientific computing (Cambridge University Press)
Savonije, G. J., \& Heemskerk, M. H. M. 1993, A\&A, 276, 409

Schwarzschild, M. 1958, Structure and evolution of the stars (Princeton: Princeton University Press)

Slettebak, A. 1982, ApJS, 50, 55

Struve, O. 1931, ApJ, 73, 94

Telting, J. H., Heemskerk, M. H. M., Henrichs, H. F., \& Savonije, G. J. 1994, A\&A, 288, 558

Townsend, R. H. D., Owocki, S. P., \& Howarth, I. D. 2004, MNRAS, 350,189

Tycner, C., Hajian, A. R., Armstrong, J. T., et al. 2004, AJ, 127, 1194

Vakili, F., Mourard, D., Stee, P., et al. 1998, A\&A, 335, 261

Waters, L. B. F. M., Cote, J., \& Lamers, H. J. G. L. M. 1987, A\&A, 185,206 